Voix et Images

voixetimages

\title{
Pour la suite du monde
}

Lucie Robert

Volume 39, numéro 2 (116), hiver 2014

URI : https://id.erudit.org/iderudit/1025197ar

DOI : https://doi.org/10.7202/1025197ar

Aller au sommaire du numéro

Éditeur(s)

Université du Québec à Montréal

ISSN

0318-9201 (imprimé)

1705-933X (numérique)

Découvrir la revue

Citer ce compte rendu

Robert, L. (2014). Compte rendu de [Pour la suite du monde]. Voix et Images, 39(2), 159-166. https://doi.org/10.7202/1025197ar

Ce document est protégé par la loi sur le droit d'auteur. L'utilisation des services d'Érudit (y compris la reproduction) est assujettie à sa politique d'utilisation que vous pouvez consulter en ligne.

https://apropos.erudit.org/fr/usagers/politique-dutilisation/
Cet article est diffusé et préservé par Érudit.

Érudit est un consortium interuniversitaire sans but lucratif composé de l’Université de Montréal, l'Université Laval et l'Université du Québec à Montréal. Il a pour mission la promotion et la valorisation de la recherche. https://www.erudit.org/fr/ 


\author{
D R A M A T U R G I E \\ Pour la suite du monde \\ $+++$
}

LUCIE ROBERT

Université du Québec à Montréal

Les auteurs dramatiques nous ont habitués aux catastrophes familiales vécues comme autant de contraintes ou limites au devenir du personnage. La référence familiale n'en finit pourtant plus de s'imposer, comme le montrent encore les pièces dont il est question ici. Après tout, $c^{\prime}$ est aujourd'hui que se créent ou se vivent les événements que l'avenir transformera en traumatismes ou en Histoire. Aussi bien les reconnaître et en disposer convenablement. Demeure néanmoins cet effet de déjà-vu créé par le retour des personnages obsédants de pères et de mères et malgré le recentrement des textes dramatiques sur les enfants (ou les très jeunes adultes) qui devront regarder en aval après avoir reçu l'héritage en amont. De même, le sujet vieillissant doit apprendre à gérer le temps, y compris celui des parents qu'il est destiné à remplacer. Peut-être en est-il ainsi des publics du théâtre, qui s'épuisent ou qui s'étiolent, dit-on. Du coup, les fictions proposées ramènent d'anciennes préoccupations sous un œil neuf, parfois hérité des textes dramatiques destinés aux jeunes publics. En ce sens, la vie apparaît comme une spirale, qui se vit en boucles, sans jamais repasser exactement au même endroit.

L'on connaît la relation étroite qui unit Evelyne de la Chenelière et le Nouveau Théâtre Expérimental. Doit-on s'étonner dès lors de la voir renouer avec l'œuvre de Marie Cardinal, dont elle a récemment adapté le roman Une vie pour deux, sous le titre $\mathrm{La}$ chair et autres fragments de l'amour ${ }^{1}$ ? Créée à l'Espace Go le 24 avril 2012, dans une mise en scène d'Alice Ronfard, la fille de Marie Cardinal, la pièce est publiée avec un avant-propos de l'auteure qui s'interroge «sur l'abondance de l'écriture dramatique contemporaine au Québec» (8) et craint, en ajoutant au lot, de se noyer elle-même dans une mer de produits culturels. Aussi entend-elle cette fois «[n]e pas écrire une nouvelle pièce de théâtre" sortie de son imagination; elle choisit "de fouiller, d'extraire, d'assembler et de répondre » (7). Toutefois, outre les accointances de nature

$$
+++
$$

1 Evelyne de la Chenelière, La chair et autres fragments de l'amour, librement inspiré du roman Une vie pour deux de Marie Cardinal, Montréal, Leméac, coll. «Théâtre/Leméac», 2012, 78 p. 
personnelle, pourquoi choisir cette auteure en particulier? Celle-ci, de l'aveu même de la Chenelière, n'est «absolument pas à la mode», ni elle comme personne, ni ses thèses sur la maternité, l'enfance, la nostalgie, la féminité, l'amour et le désamour, lesquelles s'opposent à l'action, à la violence, à la cruauté, au vide intérieur plus fréquemment travaillés sur les scènes actuelles. Sans doute faut-il voir ici un hommage autant qu'un déplacement thématique, mais sans doute aussi que l'écriture de Marie Cardinal propose des interrogations qui rejoignent l'auteure dramatique dans sa démarche artistique autant que dans sa vie privée. Car il y a une réelle parenté entre ces deux femmes, que La chair et autres fragments de l'amour permet de saisir à sa juste mesure. Et, s'il est vrai que Cardinal n'a pas écrit pour le théâtre, l'importance qu'elle accorde aux mots et au corps rejoint la matérialité de la création théâtrale.

La pièce d'Evelyne de la Chenelière est divisée en dix tableaux et met en scène un couple, Jean et Simone, en vacances dans un coin isolé de l'Irlande. C'est Simone qui a proposé ces vacances familiales dans l'espoir de reconstruire un couple dont elle sent bien qu'il se dissout peu à peu. Sans querelle et sans violence, la distance entre les deux protagonistes ne se révèle qu'aux tout petits riens de la vie quotidienne. L'action serait bien mince si Jean ne découvrait un cadavre sur la grève. C'est de cette manière que commence la pièce, sur la question de Simone : «Un cadavre? Un cadavre de quoi ? Un cadavre de qui ? » (13) On apprendra peu à peu qu'il s'agit d'une femme noyée du nom de Mary, qui a vécu à New York plusieurs années et dont plus personne n'avait eu de nouvelles depuis longtemps. Infirmière de métier, Mary s'était d'abord destinée à la médecine, et elle avait été chassée du village parce qu'elle était enceinte d'un homme marié. Elle s'était alors dirigée vers New York pour suivre ses rêves, mais, rattrapée par la vie (son fils demande plus d'attention qu'elle n'en a ; la ville est chère et dure) et par la distance (son île lui manque), elle est rentrée en Irlande. La figure de Mary, qui s'incarne en un personnage réel, dans un procédé qui emprunte au fantastique, se découvre peu à peu et souligne la distance entre Jean et Simone qui, chaque fois qu'un renseignement neuf arrive, l'interprètent diversement. Jean est séduit par le fantôme d'une femme aussi libre et autonome. Simone se retrouve à travers cette maternité qui, un jour, l'a entraînée à renoncer à ses ambitions professionnelles pour se consacrer à sa famille. Progressivement, Mary s'est infiltrée dans le quotidien de Simone: «Vous m'inventez, je vous invente en retour. [...] Je suis celle par qui vous vous découvrez. [...] Il n'y a pas que les oiseaux qui se nourrissent des morts.» (65) À mesure que l'action progresse, l'on sent le malaise croître, et c'est Mary, l'ancienne étudiante en médecine, qui pose le diagnostic: Simone est malade. Les mots s'effacent chez Simone, dont l'aphasie ne cesse de s'aggraver, avant que sa voix ne finisse par conclure:

Je ne dis plus
Je bruis
Écoute
Bientôt
Je ne saurai dire
Que
Mon amour. (78)


Il en est de cette pièce comme de plusieurs autres pièces de la Chenelière, et l'on ne sait pas toujours où tirer le trait entre le fantastique et le réalisme qui superposent deux trajectoires féminines historiquement distantes, mais partageant la même condition. Doit-on ici voir Mary comme une création de Simone qui projetterait là à la fois ses craintes et son avenir? Ou comme un personnage réellement sorti d'autrefois, dont le souvenir agirait comme révélateur des tensions du couple? L'autopsie d'une dépouille est certainement au cœur de la pièce et elle accentue la mise en scène du corps qui agit jusque sur la maladie de Simone. L'aphasie est la maladie dont souffrait également Marie Cardinal à la fin de sa vie. Simone était son deuxième prénom. «Quelle histoire, hein?» (71)

Raconter la vie prend un tout autre sens dans les textes de Simon Boudreault. $D$ pour Dieu ? ${ }^{2}$, pièce créée le 24 avril 2012, à la salle Jean-Claude-Germain du Théâtre d'Aujourd'hui, dans une mise en scène de l'auteur, repose sur deux postulats: «Dieu est mort» (9) et « [i]l paraît qu'un auteur est un Dieu pour ses personnages» (10). Ces deux propositions seront discutées à travers le récit d'une vie. L'auteur précise cependant: «Ce que j'aimerais, c'est que vous ayez l'impression que c'est le récit de votre propre vie.» (9) Au-delà des apparences que crée cette pièce à trente-deux personnages et quarante-quatre épisodes, nous sommes donc face à un texte structuré comme un conte, lequel ne trouve sa cohérence que si l'on admet la logique du récit comme fondement du dispositif scénique. Quel que soit le nombre d'acteurs en scène, il n'y a jamais qu'un seul point de vue, qui est aussi un point de fuite puisqu'il s'agit d'une vie que l'on saisit depuis le séjour dans le ventre maternel, où il fait doux et où les voix sont enveloppantes. De cet Éden originel découle la posture du narrateur qui, comme le personnage d'Amélie Nothomb autrefois (Métaphysique des tubes ${ }^{3}$ ), se croit Dieu. À partir de là, de la naissance à l'âge adulte, la vie ne sera que désillusion. De Dieu, le personnage principal déchoit progressivement au rang de fils de Dieu, puis à celui de fils d'un dieu parmi d'autres, jusqu'à découvrir que Dieu a finalement été créé par l'homme à sa ressemblance (et non l'inverse). Aussi la vie que raconte Boudreault se lit-elle comme un apprentissage en deux temps : d'abord le désenchantement par la découverte de l'imperfection du monde, puis la foi en l'avenir qui est aussi engagement envers les générations futures. Rien d'extraordinaire ne surviendra dans cette vie racontée, qui est entièrement fondée sur des événements ordinaires et communs: naissance, école, premières amitiés, divorce des parents, premier amour, première rupture jusqu'à l'engagement politique, ici vécu à travers l'intervention écologique, dont le personnage sort désabusé et vaguement toxicomane. De ce point de vue, la vie est «une prison aux barreaux acérés» (19). Le hasard d'une rencontre le ramène toutefois au salon funéraire devant le cercueil de son ami d'enfance, Éric, victime d'un accident de la route. Il y fait la connaissance de la fille d'Éric, en larmes. 
Elle lui demande s'il est Dieu puis s'endort sur ses genoux. «Je suis bien, vraiment bien. Finalement» (101), annonce le personnage, ouvrant ainsi la porte au recommencement annoncé.

Il y a quelque chose de semblable à lire dans Petite vérité inventée 4 d'Érika TremblayRoy, finaliste au prix du Gouverneur général en 2013 (la pièce de Simon Boudreault l'avait été l'année précédente), mise en lecture à la Maison-Thêâtre le 14 février 2013 sous la direction de Gill Champagne. La pièce s'ouvre en effet sur un souvenir raconté par Emma: «Quand je suis née/j’avais deux parents/C'était parfait comme ça.» (9) Nous aurons droit, encore une fois, au récit du cataclysme originel, tel que vécu dans l'enfance du personnage. Dans la pièce d'Érika Tremblay-Roy se trouve encore inscrite la logique du conte: le point de vue de la pièce sera celui d'Emma, mais d'une Emma qui doute: «Ma mémoire est surprotectrice/Elle invente [...] elle me jure qu'elle ne ment jamais/Mais moi j'arrive plus du tout à savoir laquelle de mes histoires est vraie.» (7) Le récit n'est pas linéaire, cependant, et il emprunte plutôt la forme du flash-back. En effet, au moment où le récit commence, Emma vient d'avoir un enfant. Comme son père l'avait fait autrefois, sentant elle aussi ses ailes pousser, elle quitte sa famille, emportant un vieux divan rouge, son «super radeau antinaufrage » (16), le seul objet que son père avait consenti à lui abandonner. Dans les souvenirs d'Emma, le père s'est dédoublé : le vrai est resté avec elle sous la forme d'un chat; le Clone s'est installé ailleurs et a refait sa vie, avec une Cassiopée de bonne volonté, qui «joue à la sirène et insiste pour venir me border» (20) et un nouveau petit frère: «Est-ce que je suis obligée de l'aimer?» (29) À huit ans, Emma obtient de ne plus retourner chez le Clone, où elle n'allait jamais, somme toute, qu' «en touriste» (33), et rompt d'elle-même les derniers liens qui l'unissaient à son père. Curieusement, observe-t-elle, le père chat a disparu en même temps que le Clone. Pour ses dix ans, le Clone, qui n'a pas abandonné la partie, lui offre un grand paon, un papillon épinglé dans une boîte. Elle accepte le cadeau, renoue avec ses parents, mais énonce: «je ne serai jamais un parent séparé» (47). L'avenir, on l'a vu, en a décidé autrement et Emma doit admettre: «Et ce sera à mon tour d'être clonée/Peut-être. » (50) Au même titre que chez Boudreault, l'histoire est ici répétition, comme si la condition humaine était de durer plutôt que d'agir et de transformer. L'espoir ne survit que dans le doute: «Ma mémoire est à double tranchant. [...] La vérité peut changer avec les saisons.» (51)

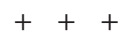

4 Érika Tremblay-Roy, Petite vérité inventée, Montréal, Dramaturges éditeurs, 2013, 51 p. 


\author{
Depuis la nuit des temps, \\ le monde est fasciné par le ciel. \\ Les hommes ont toujours caressé le rêve \\ de s'envoler comme les oiseaux. (7)
}

Sans doute faut-il voir là l'intérêt que portent les jeunes auteurs dramatiques à la métaphore du papillon, que Marie-Ëve Huot, directrice du Théâtre Ébouriffé, reprend ici dans Nouds papillon ${ }^{5}$, sa première pièce, créée le 2 mai 2013 à Laval dans le cadre des Rencontres Théâtre Ados. Dominent encore ici la figure du père et la problématique de l'héritage qu'il transmet à sa fille, Amélie, onze ans. Au début de la pièce, Amélie, qui collectionne les papillons, doit préparer un exposé scolaire sur Le métier que je voudrais faire plus tard. Au moment de présenter cet exposé, elle apprend la mort de son père, victime d'un écrasement d'avion en Gaspésie, et elle se mure dans le silence. Aussi la pièce sera-t-elle d'abord l'écriture de ses pensées, de ses souvenirs, racontés par elle néanmoins: "Je suis dans tous mes états. » (10) L'accompagne une sorte de double, Amelia, souvenir d'Amelia Earheart, l'aviatrice, qui lui sert de destinataire imaginaire, et qui lui permet de réapprendre à vivre. Amélie est d'ailleurs née le jour anniversaire de l'arrivée d'Amelia Earheart à Londres, et le travail de l'accouchement avait duré exactement le même temps que la traversée de l'aviatrice. Elle se rappelle ainsi le projet que son père avait été invité à présenter dans sa classe à elle : devenir pilote d'avion. La présentation se prolongeait dans le rappel des grands héros de l'aviation: les frères Wright, Léonard de Vinci, Charles Lindbergh et Amelia Earheart. Souffrant de vertige, il n'est jamais devenu pilote. Néanmoins, ce jour-là, «quand papa est rentré à la maison/il avait l'air plus jeune que moi» (25). Il avait renoué avec ses carnets d'enfant et ses avions-jouets. D'autres souvenirs viendront ponctuer le récit d'Amélie: les cartes postales aux adresses de retour imaginaires, choisies dans le monde entier, par exemple.

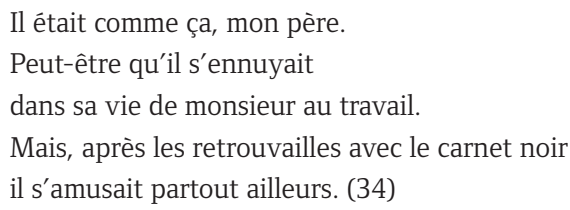

Après l'accident d'avion, Amélie doit ainsi réapprendre à vivre avec l'absence. Au cours de l'année - l'action de la pièce dure une année - , elle découvre l'histoire de Chu Ta, un peintre chinois qui s'est muré dans le silence quand les Mandchous ont pris le pouvoir, assassiné sa famille et interdit sa peinture. Il s'est alors réfugié dans sa montagne et a peint tout ce qu'il voyait. Amélie comprend l'histoire, qu'elle fera sienne. Le jour de ses douze ans, ses premiers mots seront pour demander un babillard où épingler ses papillons. Ce sera là sa passion à elle.

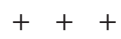

5 Marie-Ėve Huot, Nœuds papillon, Carnières, Lansman éditeur, 2013, 45 p. 
Une pièce de Lise Vaillancourt est chaque fois un événement, bien qu'elle ait écrit plus souvent pour les jeunes publics (on se rappellera Le petit dragon et La balade de Fannie et Carcassonne ${ }^{6}$ ) que pour les publics adultes. La corneille ${ }^{7}$, créée au Thêâtre du Rideau Vert le 18 septembre 2012, dans une mise en scène de Geoffrey Gaquère, est sa première incursion sur la scène grand public, elle qui est une ancienne du Théâtre expérimental des femmes et l'une des fondatrices de l'Espace Go, normalement plus proche de l'esthétique de la Chenelière et de Ronfard que de celle de Denise Filiatrault. La corneille, qui revient sur la figure de la mère, contraste avec les trois pièces précédentes, centrées sur la figure du père. Il y a sans doute là un effet de génération qui se lit aussi dans une autre forme de contraste en ce que, comme Evelyne de la Chenelière, Vaillancourt emploie des éléments de fantastique plutôt que la logique du conte. Non que le conte soit tout à fait absent de la pièce puisque Julie s'adresse d'emblée au public: "Ça a commencé comme ça; en revenant de mon cours de yoga, une corneille volait dans mon loft. » (7) Nous sommes six mois après la mort du père et voilà que la mère, verbomotrice, culpabilisante, exubérante, s'annonce. Or, il y a vingt-cinq ans que Julie l'invite sans succès. Cette arrivée impromptue suscite donc quelques inquiétudes: la présence d'une femme plus âgée, qui cuisine, ne fréquente pas les voisins, vit entourée de monde qu'elle ne veut pas voir, sans doute profondément désemparée de la mort de son mari, n'est pas l'événement heureux que souhaiterait Julie, quarante-huit ans, célibataire à l'existence rangée entre le crack et la tisane bio, sans vie sociale connue, et qui admet ne pas aimer sa mère, bien qu'elle paraisse en même temps désireuse de se rapprocher d'elle. Dans ce contexte, la présence de la mère agit comme un élément perturbateur. Au matin de la deuxième journée, elle a déjà acheté le pain, sorti les poubelles (où traînait un cadeau offert à sa fille, jamais déballé), vendu son appartement (qui, de toute manière, avait été acheté pour les maitresses du mari) et pris des arrangements pour trouver un condo en Floride. Toutefois, la situation est moins claire qu'elle ne le paraît, car Julie, qui entretient des relations amicales avec sa voisine Millie, remarque que, chaque fois que Millie entre, sa mère disparaît. Après le départ de Millie, elle la retrouve dans les endroits les plus incongrus: recroquevillée derrière la cuisinière, dans la baignoire, dans le frigo (assise sur les brocolis), etc. Pas moyen de l'en sortir. Julie raconte encore: «Les jours suivants, ça a continué» (25), et elle admet: «J'ai fini par m'habituer à sa présence, mais surtout à ses absences» (27), jusqu'à vouloir éclaircir «ça, la mort de mon père» (28). Elle s'offre alors une tournée dans les bars de la rue Saint-Laurent et conclut: «il faut faire la paix avec ce qu'on ne comprend pas» (37). Au retour, Julie apprend la mort de sa mère au moment même où la corneille tente de sortir d'un couloir qui la retient prisonnière. Cette corneille, c'était donc la mère aussi, qui peut s'envoler maintenant que sa fille est rassurée sur ses sentiments. Millie, qui vient ellemême de vivre une rupture amoureuse, reste avec Julie... pour respirer.

6 Lise Vaillancourt, Le petit dragon; La balade de Fannie et Carcassonne, Carnières, Lansman, 1999, 46 p. 7 Lise Vaillancourt, La corneille, Montréal, Leméac, coll. "Théâtre/Leméac», 2012, 47 p. 
"Qui commence, qui donne suite? Doit-on croire que l'une dirige et que l'autre exécute? » (7) demande Martine Beaulne. Celle-ci avait déjà publié Le passeur d'âme ${ }^{8}$ en 2004. De Sylvie Drapeau, outre quelques brefs entretiens de promotion, nous n'avons jamais eu de réflexion approfondie qui permette d'appréhender la nature de son travail. Aussi faut-il saisir l'occasion de feuilleter Voir de l'intérieur ${ }^{9}$, dialogue entre une comédienne et une metteure en scène, comme une vraie conversation sur le théâtre, comme une pièce à deux voix, intimiste et généreuse. L'idée du livre serait née à bord d'un avion entre Montréal et Vancouver lors de la tournée d'Avaler la mer et les poissons ${ }^{10}$ (de Sylvie Drapeau et Isabelle Vincent) en 2007. Les auteures précisent : «Ces échanges, nous avons voulu les rendre publics dans l'espoir de susciter d'autres réflexions et de poursuivre un questionnement sur cet art théâtral qui nous passionne.» (11) Au fil des pages se découvre d'abord le malentendu à la source même du travail thêâtral. L'acteur, en effet, croit que le metteur en scène sait ce qu'il va faire. Le metteur en scène découvre à travers l'acteur ce que sera la matérialité de sa réalisation (au-delà de l'analyse dramaturgique de la pièce), puisque l'acteur est d'abord le personnage. «Tout l'être tend vers le personnage, vers les mots qui veulent l'incarner. » (20) Le corps est donc le point de départ, ce que dit le mot «incarner». C'est au metteur en scène de «reconnaître les bons corps» (27) et de les harmoniser entre eux. La conversation suit ensuite assez librement un certain nombre de méandres, où les auteures explorent les enjeux du travail : le choix du répertoire, la confusion possible entre l'identité de l'acteur et celle du personnage, les moments d'incertitude, imprévisibles mais inévitables, le travail qui met le corps en jeu comme s'il s'agissait d'un instrument de musique et la nécessaire désobéissance aux consignes: «Il faut être soi-même un peu metteur en scène pour bien désobéir.» (52) La metteure en scène adhère à cette proposition, et elle ajoute : «Il faut obéir à ses instincts, avoir le courage de nommer le désarroi, l'incohérence, le factice.» (53) Beaulne et Drapeau ont en commun de concevoir la création théâtrale comme un acte collectif, comme la rencontre de plusieurs artistes qui enrichissent la proposition scénique du metteur en scène: « J'attends d'un acteur qu'il apporte un point de vue personnel par son interprétation. » (85) Le travail de création leur apparaît comme un voyage à la manière de ce qu'en écrivait Gabrielle Roy dans La détresse et l'enchantement ${ }^{11}$. On sent d'ailleurs une certaine détresse devant les conditions désormais dévolues au travail thêâtral: réduction du temps de répétition, production trop rapide, maximum de vingt-quatre représentations par pièce, jamais plus. «Le fait de devoir aller si vite, de devoir être si efficaces, si productifs [...], tout cela enlève de la valeur [...], cela banalise les productions: ce n'est qu'une autre production dans cette succession de spectacles. C'est le fast thêâtre. » (100) Suivra le retour sur l'expérience de l'écriture, à propos d'Avaler

$$
++
$$

8 Martine Beaulne, Le passeur d'âme. Genèse et métaphysique d'une écriture scénique, Montréal, Leméac, 2004, 199 p. 9 Martine Beaulne et Sylvie Drapeau, Voir de l'intérieur, Montréal, Dramaturges éditeurs, coll. «Didascalies», 2012, 141 p. 10 Sylvie Drapeau et Isabelle Vincent, Avaler la mer et les poissons, Montréal, Dramaturges éditeurs, 2007, 89 p. 11 Gabrielle Roy, La détresse et l'enchantement, Montréal, Boréal Express, $1984,505 \mathrm{p}$. 
la mer et les poissons, et sur l'expérience personnelle que représente le travail sur des textes dramatiques, au croisement des personnages et de la personne. Visiblement, ces deux femmes ont rencontré dans leurs échanges un bonheur d'expression qui ne trouve son parallèle que dans l'unité de leur projet et dans la passion qui les habite. À la lecture, ce bonheur s'éprouve aussi dans sa plénitude. 